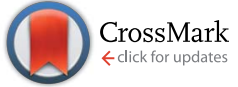

Cite this: RSC Adv., 2017, 7, 11684

Received 18th November 2016 Accepted 10th February 2017

DOI: 10.1039/c6ra26998b

rsc.li/rsc-advances

\section{Recyclable palladium-graphene nanocomposite catalysts containing ionic polymers: efficient Suzuki coupling reactions $\uparrow$}

\author{
Tae Hui Kwon, $\ddagger^{a}$ Kie Yong Cho, $\$^{\text {bc }}$ Kyung-Youl Baek, ${ }^{c}$ Ho Gyu Yoon ${ }^{\star b}$ \\ and B. Moon Kim*a
}

\begin{abstract}
Palladium nanoparticles on ionic polymer-doped graphene (Pd-IPG) nanocomposite catalysts have been investigated for efficient Suzuki coupling reactions. This combination effected highly accelerated Suzuki coupling reactions due to several advantageous features associated with the flanking ionic polymer part of the catalyst system. These include a high level of Pd incorporation, excellent dispersion stability, and increased accessibility and diffusion of the substrates onto the surface of Pd NPs. The enhanced availability of the Pd catalyst to the reacting substrates is believed to allow for ca. 16-fold higher catalytic activity than that of $\mathrm{Pd}$-graphene without the ionic polymer. Moreover, high recycling capability of the catalyst (10 times) in combination with excellent product yields ( $>96 \%)$ and no significant leaching of the catalyst upon hot-filtration test suggest that the Pd-IPG nanocomposite catalysts have high reusability with significant retention (>95\%) of the Pd species.
\end{abstract}

\section{Introduction}

Palladium-catalyzed Suzuki-coupling transformations constitute a powerful methodology for the formation of carboncarbon $(\mathrm{C}-\mathrm{C})$ bonds. It features several beneficial points such as high convergence in synthetic strategy, tolerance to a broad range of functional groups, insensitivity towards water, and generally high regio- and stereo-selectivities. ${ }^{1-3}$ To date, the Suzuki-coupling reactions have been conventionally performed primarily through the use of a homogeneous Pd catalysts. ${ }^{4,5}$ However, Pd-catalyzed homogeneous reactions have suffered from limitations such as the requirement of toxic and airsensitive phosphine ligands, safety issues of products due to metal contamination and difficulties in catalyst recovery and recyclability. ${ }^{6,7}$ Tireless efforts toward alleviating these shortcomings have continued and development of heterogeneous catalytic systems employing various supports to Pd nanoparticles (NPs) came as one remedy favorably leading to effective retaining of catalytic activity and promoting durability of

\footnotetext{
${ }^{a}$ Department of Chemistry, Seoul National University, Seoul 08826, Republic of Korea. E-mail:kimbm@snu.ac.kr

${ }^{b}$ Department of Materials Science and Engineering, Korea University, Seoul 02841, Republic of Korea. E-mail: hgyoon@korea.ac.kr

${ }^{c}$ Materials Architecturing Research Center, Korea Institute of Science and Technology, Seoul 02792, Republic of Korea

$\dagger$ Electronic supplementary information (ESI) available: Detailed experimental procedures and calculations, Tables S1 and S2, Schemes S1 and S2, Fig. S1-S5. See DOI: $10.1039 / \mathrm{c} 6 \mathrm{ra} 26998 \mathrm{~b}$

$\ddagger$ These authors contributed equally to this work.
}

the catalyst because of maximized active surface area and enhanced anchoring ability. ${ }^{8-11}$

For desirable catalytic performance of heterogeneous Pd catalysts, various carbon supports of Pd NPs have been developed, such as functionalized carbon nanotubes (CNTs), graphenes and their combinations. ${ }^{12-20}$ These approaches have been somewhat successful in solving the issues associated with the homogenous catalysis system, however, the limited functional groups on the carbon supports often caused detrimental aggregation through long range $\pi-\pi$ stacking and weak interfacial interaction with Pd NPs, leading to low quality incorporation, easy dissolution and loss of Pd NPs from the support, and ambiguous dispersion stability. ${ }^{21-23}$

Recently efforts to overcome these limitations have emerged through employment of non-covalently functionalized carbon supports equipped with polymer compatibilizers. ${ }^{24-27}$ These systems allowed for a great nanocomposite catalyst with uniform and well-distributed Pd NPs on the carbon supports as well as noticeably enhanced catalytic performance due to better dispersion property, strong interfacial linking, and adequate interaction with substrates. ${ }^{10,27-31}$ Specifically, in our recent report, the ionic polymer-doped graphene support was explored to develop effective catalysts for hydrogenation reaction through combination with fine-sized Pd NPs (1-3 nm), resulting in the ultra-accelerated catalytic reduction of methylene blue. Effective interaction and diffusion of reacting species onto the surface of Pd NPs along with its desirable dispersion property in the aqueous medium were believed to be the main reason for the successful catalytic activity. ${ }^{29}$ However, the fine-sized metal NPs can be generally less stable under harsh reaction 
conditions, such as high temperature and the presence of a strong base. ${ }^{32}$

Herein, we demonstrate that the combination of multiscaled Pd NPs with ionic polymer-doped reduced graphene oxide (rGO) supports (Pd-IPG) culminated in markedly enhanced catalytic activity (TOF: $990 \mathrm{~h}^{-1}$ ) and yield (>99\%) in the Suzuki coupling reaction between iodobenzene and phenylboronic acid in comparison to that of the catalyst without the ionic polymer (Pd-rGO). The Pd-IPG nanocomposite catalysts showed good recyclability during 10-time repeated cycles.

\section{Experimental section}

\subsection{Synthesis of Pd-IPG nanocomposite catalysts}

Pyrene functionalized poly(dimethylaminoethyl methacrylate)$b$-poly[(ethylene glycol) methyl ether methacrylate] (PyPDMAEMA- $b$-PPEGMEMA), quaternary ammonium cation containing ionic polymer-doped reduced graphene oxide $(0.4 \mathrm{mg}$, IPG), prepared following our previous report, was dispersed in $15 \mathrm{~mL}$ DMF by sonication for $10 \mathrm{~min} \cdot{ }^{31}$ Benzoic acid $(7.3 \mathrm{mg}$, Sigma-Aldrich, $>99.5 \%$ ) was sequentially added to the IPG solution, and the mixture was gently sonicated for $5 \mathrm{~min}$. Thereafter, this solution was placed on an ice bath and agitated for $5 \mathrm{~min}$. After the foregoing procedure, aq. $\mathrm{Na}_{2} \mathrm{PdCl}_{4}$ solution ( $2 \mathrm{~mL}, 20 \mathrm{mM}$ in water, Sigma-Aldrich, 99.995\%) was injected to the prepared solution, and the mixture was strongly agitated in an ice bath for $10 \mathrm{~s}$. The reaction was retained at $130{ }^{\circ} \mathrm{C}$ for $30 \mathrm{~min}$. Thereafter, the reaction solution was stirred in an ice bath for $10 \mathrm{~min}$. The crude product was purified by centrifugation 3 times using an ethanol and acetone $(2: 1)$ mixture. The obtained precipitates were dispersed in $100 \mathrm{~mL}$ of THF by sonication for $10 \mathrm{~min}$ and the resulting products were obtained by filtration under reduced pressure. The filtered powder was dried at room temperature under reduced pressure for $24 \mathrm{~h}$.

\subsection{Evaluation of catalytic activity of the Pd-IPG nanocomposite catalysts for Suzuki coupling reactions}

Suzuki coupling reactions using the Pd-IPG were performed at $60{ }^{\circ} \mathrm{C}$ after preparing the reaction solution including arylboronic acid $(0.12 \mathrm{mmol})$, aryl iodide $(0.1 \mathrm{mmol}), \mathrm{NaOH}(0.20$ $\mathrm{mmol})$, anisole $(0.1 \mathrm{mmol}), \mathrm{Pd}-\mathrm{IPG}(0.1 \mathrm{~mol} \%)$ in ethanol/water $(1: 1 \mathrm{v} / \mathrm{v}, 0.8 \mathrm{~mL})$. The reaction yield was monitored by GC analysis, where a small amount of the crude solution was taken and applied to GC analysis at the predetermined time after simple filtration. The completed reaction mixture was cooled to room temperature. Thereafter, Pd-IPG was separated from the reaction by facile filtration and the filtrate was extracted with ethyl acetate. The obtained organic part was used for GC analysis. The reaction yield was calculated by using anisole as an internal standard.

\subsection{Recyclability test of the Pd-IPG nanocomposite catalysts}

Suzuki coupling reactions of phenylboronic acid $(0.60 \mathrm{mmol})$ with iodobenzene $(0.50 \mathrm{mmol})$ were performed using Pd-IPG (0.1 mol\%), $\mathrm{NaOH}(1.0 \mathrm{mmol})$, and anisole $(0.50 \mathrm{mmol})$ in ethanol/water $(1: 1 \mathrm{v} / \mathrm{v}, 4.0 \mathrm{~mL})$. After dilution of the crude reaction solution with ethyl acetate, the solution was filtered to separate the Pd-IPG. The acquired Pd-IPG was redispersed in ethanol/water $(1: 1 \mathrm{v} / \mathrm{v}, 4.0 \mathrm{~mL})$ for the next reaction and the organic part was used for GC analysis to calculate the reaction yield after extraction with ethyl acetate. The foregoing procedure was repeated up to ten times to investigate the recyclability of Pd-IPG.

\subsection{Hot-filtration test of the Pd-IPG nanocomposite catalysts}

For hot-filtration test, a Suzuki coupling reaction between phenylboronic acid $(0.24 \mathrm{mmol})$ and iodobenzene $(0.20 \mathrm{mmol})$ was conducted under optimized conditions. The reaction was maintained for $30 \mathrm{~min}$ and then a half volume of the reaction solution was taken and filtered while hot. Thereafter, the reaction yields of the hot filtrate and an unfiltered half of the solution were monitored through GC analysis after sampling small amounts of the reaction solutions at the predetermined time.

\subsection{Characterizations}

Scanning transmission electron microscopy (STEM) was applied for analysis of elemental mapping and energy dispersive X-ray (EDX) (FEI Talos F200X, maximum accelerating voltage of $200 \mathrm{kV}$ ) analyses. X-ray photoelectron spectroscopy (XPS) analysis was performed under reduced pressure using an X-ray photoelectron spectrometer (X-TOOL, ULVAC-PHI) with a monochromatic AlK $\alpha$ source. The phase was identified by matching each characteristic peak with the JCPDS files. Synthesized graphene supports were characterized using Raman spectrometer (LabRam ARAMIS IR2, Horiba, Japan). Xray diffraction patterns were acquired on a Rigaku diffractometer (Rigaku Smart Lab, Rigaku Co., Japan) operated at $45 \mathrm{kV}$ and $40 \mathrm{~mA}$ with $\mathrm{CuK} \alpha$ radiation $(\lambda=1.5406 \AA)$ using a diffracted beam monochromator. Data were collected between $2 \theta=5^{\circ}$ and $100^{\circ}$ at $0.01^{\circ}$ intervals. Evaluation of yields and catalytic activity was determined by gas chromatography (GC, Hewlett Packard 5890) analysis. ${ }^{1} \mathrm{H}$ and ${ }^{13} \mathrm{C}$ NMR spectra were obtained on Agilent MR DD2 (400 MHz) spectrophotometer.

\section{Results and discussion}

The multi-scaled Pd NPs loaded on the pyrene functionalized poly(dimethylaminoethyl methacrylate)-b-poly[(ethylene glycol) methyl ether methacrylate] ionic polymer-doped graphene support (Pd-IPG) were synthesized by a facile in situ method (Schemes S1 and S2 $\dagger$ ), which used sodium tetrachloropalladate as a metal precursor, benzoic acid (BA) as a structure-directing agent, $N, N$-dimethyl formamide (DMF) as a solvent and a reducing agent. For comparison, Pd NPs loaded rGO without the ionic polymer (Pd-rGO, Pd: $42.7 \mathrm{wt} \%$ ) was synthesized using the same conditions for the synthesis of the Pd-IPG and characterized as shown in the ESI (Fig. S1 $\dagger$ ).

The acquired Pd-IPG nanocomposite catalysts were characterized through scanning transmission electron microscopy (STEM), which exhibited well-distributed multi-scaled Pd NPs 
on the IPG (Fig. 1A). STEM energy-dispersive X-ray (STEM-EDX), powder X-ray diffraction (XRD), and X-ray photoelectron spectroscopy (XPS) analyses of Pd-IPG were performed for the investigation of the composition and structure of Pd NPs on IPG. The STEM-EDX elemental mapping of Pd-IPG showed that the observed NPs comprised Pd atoms, and the STEM-EDX spectrum exhibited 49.7 wt $\%$ of $\mathrm{Pd}$ relative to $\mathrm{C}, \mathrm{O}$, and $\mathrm{N}$ contents originated from IPG (Fig. 1B and C, respectively).

The XRD patterns of Pd-IPG showed predominant peaks at $25.1,39.5,45.9,67.0^{\circ}$ corresponding to the face-centered cubic (FCC) structure, except for the broad peak at $25.1^{\circ}$, which can be
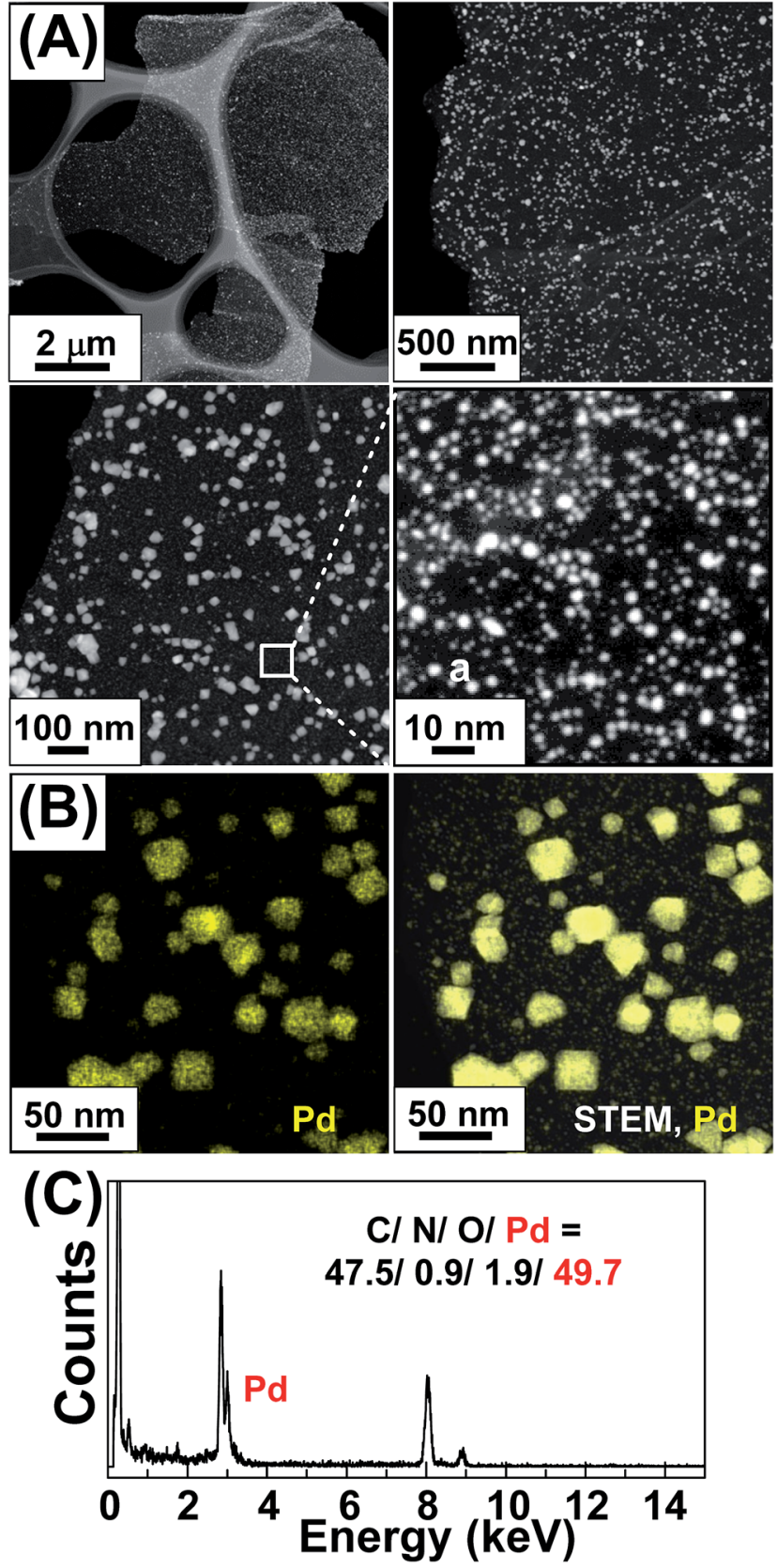

Fig. 1 (A) STEM images (inset (a): magnified image for small-sized Pd NPs, size distribution 3-5 $\mathrm{nm}$ ), (B) STEM-EDX elemental mapping images for Pd, and (C) STEM-EDX spectrum of Pd-IPG. originated from the IPG carbon support (Fig. 2A). ${ }^{\mathbf{1 0 , 3 1}}$ Moreover, the average crystalline size of Pd NPs on IPG was evaluated to be $8.4 \mathrm{~nm}$ from the XRD pattern with the relation of Scherrer formula, which was in good agreement with corresponding STEM analysis exhibiting the broad distribution of Pd NPs in a size (large numbers: $2-5 \mathrm{~nm}$, small numbers: $15-30 \mathrm{~nm}$ ), as described in the ESI (eqn (S1) and Table S1†). ${ }^{\mathbf{1 0}}$ The deconvoluted XPS Pd 3d spectra of Pd-IPG showed predominant peaks at $334.7 / 340.0 \mathrm{eV}$ and 336.0/340.9 eV corresponding to $\mathrm{Pd}^{0}$ and $\mathrm{Pd}^{2+}$, respectively (Fig. 2B). ${ }^{16}$ The XPS result indicated that the Pd NPs in Pd-IPG mainly comprises the metallic state Pd.

The interfacial interaction of three components including rGO, ionic polymer, and Pd NPs in Pd-IPG was characterized through Raman spectroscopy (Fig. 2C). Due to the presence of the ionic polymer to rGO, the predominant peaks of the rGO Raman spectrum at 1339.1 and $1582.5 \mathrm{~cm}^{-1}$ corresponding to D and $\mathrm{G}$ bands were shifted to higher wavenumbers to 1347.1 and $1585.4 \mathrm{~cm}^{-1}$, respectively. After incorporation of IPG with Pd NPs, these two bands were also slightly shifted to 1348.2 and $1589.6 \mathrm{~cm}^{-1}$. These positive Raman shifts can be explained by the influence of intermolecular electron-transfer, indicating the well-developed intercalation structure. ${ }^{29}$

To optimize the catalytic reaction conditions of Pd-IPG, Suzuki coupling reactions using iodobenzene and phenylboronic acid as model substrates were screened with regard to various bases and solvents (Table 1). The reactions were first screened with various bases at $60{ }^{\circ} \mathrm{C}$ with $0.3 \mathrm{~mol} \%$ Pd-IPG in ethanol/water ( $\mathrm{v} / \mathrm{v}=1: 1$ ) (Table 1 , entries 1-6). As shown, reactions using most inorganic bases proceeded furnishing high yields ( $>98 \%)$ except for that with $\mathrm{KHCO}_{3}(81 \%)$, indicating
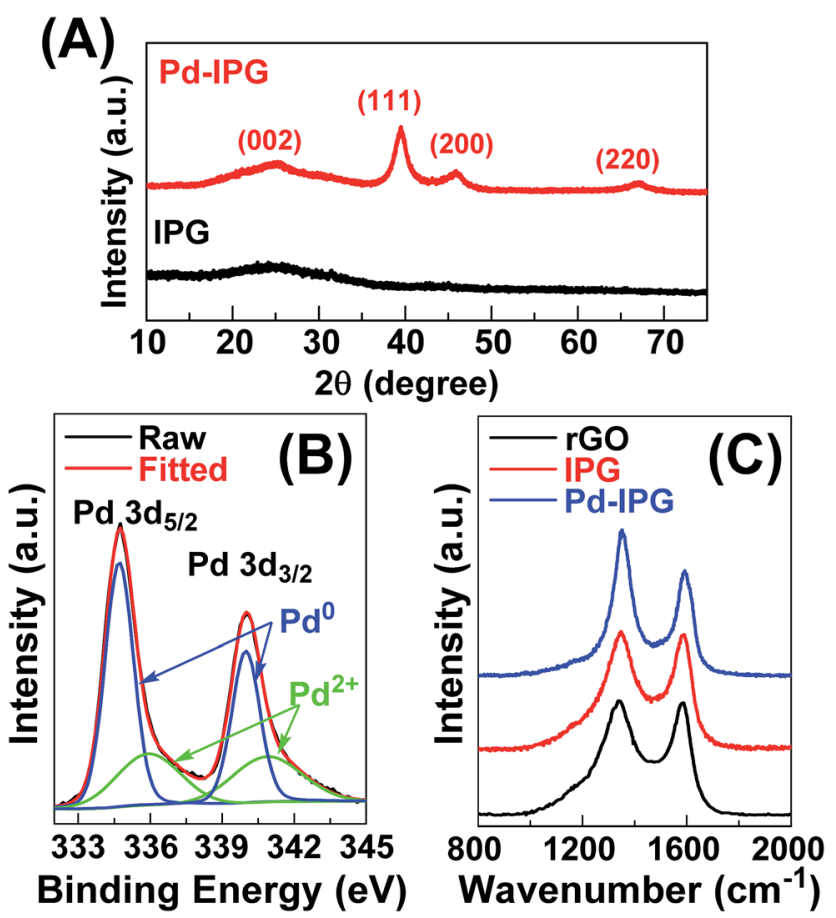

Fig. 2 (A) Powder XRD patterns of IPG and Pd-IPG. (B) XPS Pd 3d spectrum of Pd-IPG. (C) Raman spectra of rGO, IPG, and Pd-IPG. 
Table 1 Optimization of the reaction conditions for Suzuki coupling reactions between iodobenzene and phenylboronic acid catalyzed by the Pd-IPG nanocomposite catalysts ${ }^{a}$

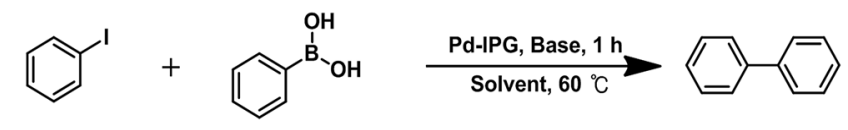

\begin{tabular}{|c|c|c|c|c|}
\hline Entry & Solvent & Base & Cat. (mol\%) & Yield $^{b}(\%)$ \\
\hline 1 & EtOH $: \mathrm{H}_{2} \mathrm{O}=1: 1$ & TEA & 0.3 & 51 \\
\hline 2 & EtOH $: \mathrm{H}_{2} \mathrm{O}=1: 1$ & $\mathrm{KHCO}_{3}$ & 0.3 & 81 \\
\hline 3 & EtOH $: \mathrm{H}_{2} \mathrm{O}=1: 1$ & $\mathrm{~K}_{2} \mathrm{CO}_{3}$ & 0.3 & 98 \\
\hline 4 & EtOH $: \mathrm{H}_{2} \mathrm{O}=1: 1$ & $\mathrm{Cs}_{2} \mathrm{CO}_{3}$ & 0.3 & 98 \\
\hline 5 & EtOH $: \mathrm{H}_{2} \mathrm{O}=1: 1$ & $\mathrm{Na}_{2} \mathrm{CO}_{3}$ & 0.3 & 99 \\
\hline 6 & EtOH $: \mathrm{H}_{2} \mathrm{O}=1: 1$ & $\mathrm{NaOH}$ & 0.3 & $>99$ \\
\hline 7 & EtOH $: \mathrm{H}_{2} \mathrm{O}=1: 1$ & $\mathrm{NaOH}$ & 0.2 & 99 \\
\hline 8 & EtOH $: \mathrm{H}_{2} \mathrm{O}=1: 1$ & $\mathrm{NaOH}$ & 0.1 & 99 \\
\hline 9 & EtOH $: \mathrm{H}_{2} \mathrm{O}=1: 1$ & $\mathrm{Na}_{2} \mathrm{CO}_{3}$ & 0.1 & 60 \\
\hline 10 & $\mathrm{H}_{2} \mathrm{O}$ & $\mathrm{NaOH}$ & 0.1 & 20 \\
\hline 11 & $\mathrm{MeOH}$ & $\mathrm{NaOH}$ & 0.1 & 83 \\
\hline 12 & EtOH & $\mathrm{NaOH}$ & 0.1 & 86 \\
\hline 13 & $\mathrm{CH}_{3} \mathrm{CN}$ & $\mathrm{NaOH}$ & 0.1 & 1 \\
\hline 14 & DMF & $\mathrm{NaOH}$ & 0.1 & NR \\
\hline 15 & THF & $\mathrm{NaOH}$ & 0.1 & NR \\
\hline 16 & DMF $: \mathrm{H}_{2} \mathrm{O}=1: 1$ & $\mathrm{NaOH}$ & 0.1 & 50 \\
\hline 17 & THF $: \mathrm{H}_{2} \mathrm{O}=1: 1$ & $\mathrm{NaOH}$ & 0.1 & 48 \\
\hline
\end{tabular}

${ }^{a}$ Reaction conditions: iodobenzene $(0.1 \mathrm{mmol})$, phenylboronic acid $(0.12 \mathrm{mmol})$, base $(0.2 \mathrm{mmol})$, and solvent $(0.8 \mathrm{~mL})$ at $60{ }^{\circ} \mathrm{C}$ under air. ${ }^{b}$ Yields were calculated from GC analysis with anisole as an internal standard.

that strong bases are more effective than weak ones. Whereas, TEA, an organic base, was much less effective in this system. To identify the best base for this system, the content of Pd-IPG was reduced from $0.3 \mathrm{~mol} \%$ to $0.1 \mathrm{~mol} \%$, and $\mathrm{NaOH}$ still showed sustained reactivity with high yield (99\%). However, $\mathrm{Na}_{2} \mathrm{CO}_{3}$ showed noticeably reduced yield from $99 \%$ to $60 \%$ after the catalyst content was decreased to $0.1 \mathrm{~mol} \%$. From the base screening tests, the most optimal Suzuki coupling reaction with Pd-IPG was achieved through the use of $\mathrm{NaOH}$.

With the $\mathrm{NaOH}$ in hand as an optimal base for this system, representative protic $\left(\mathrm{H}_{2} \mathrm{O}, \mathrm{MeOH}, \mathrm{EtOH}\right)$ and aprotic solvents $\left(\mathrm{CH}_{3} \mathrm{CN}, \mathrm{THF}\right)$ were screened for the reaction with Pd-IPG. Reactions in protic solvents, except for water, proved to proceed with much better reactivity in comparison to those in aprotic ones (Table 1, entries 11 and 12 vs. 13-15, respectively). This result could be attributed to the poor solubility of $\mathrm{NaOH}$ in aprotic solvents. To confirm the solubility issue of $\mathrm{NaOH}$ in aprotic solvents, aqueous solutions of either THF or DMF were applied for the Suzuki coupling reaction and the results clearly supported the proposed reason for low reactivity in the cases of the aprotic solvents (Table 1, entries 16 and 17). Meanwhile, the use of water only as a solvent produced only $20 \%$ yield despite good solubility of $\mathrm{NaOH}$, presumably due to the poor solubility of the organic substrates in water. As such, the Suzuki coupling reaction at $60{ }^{\circ} \mathrm{C}$ using $\mathrm{NaOH}$ as a base in $\mathrm{EtOH} / \mathrm{H}_{2} \mathrm{O}(\mathrm{v} / \mathrm{v}=1: 1)$ as a solvent was selected as an optimized condition for the reaction employing $0.1 \mathrm{~mol} \%$ of Pd-IPG.
The optimized reaction protocol for the Suzuki coupling reaction between various substituted aryl halides and aryl boronic acids was applied to evaluate the catalytic activity of PdIPG using Pd-rGO as a reference catalyst, and corresponding yields and turnover frequency (TOF) values were summarized in Table 2 . The use of Pd-IPG $(0.1 \mathrm{~mol} \%)$ in the coupling reaction between iodobenzene and phenylboronic acid showed high catalytic activity, with 99\% yield in $1 \mathrm{~h}$ and the TOF value of 990 $\mathrm{h}^{-1}$, and the reactivity employing the Pd-IPG catalyst was largely dictated by the electronic nature of substituents in aryl iodides; aryl iodides with an electron withdrawing substituent showed higher catalytic activity relative to those with electrondonating substituents. Specifically, the reaction with aryl iodide having an acetyl substituent showed 2-fold higher TOF value $\left(2000 \mathrm{~h}^{-1}\right)$ for the coupling product than that of iodobenzene, and achieved 8-fold higher TOF value in comparison to the corresponding hydroxyl-substituted aryl iodide $\left(245 \mathrm{~h}^{-1}\right.$, Table 2, entries 1, 2 and 4, respectively).

It is intriguing to note that for the production of 4-methoxy1,1'-biphenyl the reaction between 4-methoxyphenylboronic acid and phenyl iodide (entry 5 ) is a much better choice with 3fold higher TOF value $\left(970 \mathrm{~h}^{-1}\right)$ compared to that of 4 methoxyphenyl iodide and phenylboronic acid (entry 3). However, the reaction of 4-hydroxyphenylboronic acid led to a considerable decrease in the reactivity with iodobenzene (Table 2, entry 6). These results indicate that the rates of coupling reactions using Pd-IPG are quite sensitive to the

Table 2 Evaluation of catalytic activity of Pd-IPG and Pd-rGO according to the various substrates under optimized conditions ${ }^{a}$

$\begin{array}{llllllll} & & & & & \\ \end{array}$

${ }^{a}$ Reaction conditions: aryl halide $(0.1 \mathrm{mmol})$, arylboronic acid (0.12 $\mathrm{mmol}), \mathrm{NaOH}(0.2 \mathrm{mmol})$, and $\mathrm{EtOH} / \mathrm{H}_{2} \mathrm{O}(0.8 \mathrm{~mL})$ at $60{ }^{\circ} \mathrm{C}$ under air. ${ }^{b}$ GC yield were calculated by using anisole as an internal standard. ${ }^{c}$ TOF value was calculated by moles of product with per mole of Pd catalyst per hour. ${ }^{d}$ Pd-IPG (0.1 mol\% to aryl halide). ${ }^{e}$ Pd-rGO (0.5 mol\% to aryl halide). 
electronic nature of the substrates and the selection of appropriate substrates is necessary for more effective coupling reactions.

To explore the ionic polymer doping effect, the Suzuki coupling reactions with the same substrates employing Pd-rGO as a reference catalyst were examined (Table 2, entries 9-16). Since the reactions employing $0.1 \mathrm{~mol} \% \mathrm{Pd}-\mathrm{rGO}$ required significantly long reaction time (over $24 \mathrm{~h}$ ), $0.5 \mathrm{~mol} \% \mathrm{Pd}-\mathrm{rGO}$ was employed for the comparison reaction. The activity profile of Pd-rGO with respect to the electronic nature of the substrates including aryl bromides was fully identical to that of the corresponding Pd-IPG. However, the catalytic activities of Pd-rGO were significantly lower for all substrates than that of Pd-IPG, exhibiting 21-fold maximum TOF reduction in the case of entry 10 compared to entry 2 (Table 2 ).

The Suzuki coupling reactions with bromide substrates were performed to evaluate the comparative reactivity of the Pd-IPG to the Pd-rGO as a reference catalyst (Table 2, entries 7, 8, 15, and 16). The improved reactivity of Pd-IPG based on the ionic polymer doping effect was also prominent in the case of reactions with bromide substrates, specifically furnishing almost 9fold maximum enhancement in the reaction of acetylsubstituted aryl bromide with phenylboronic acid (Table 2, entries 8 and 16). However, the TOF values in the reaction with bromide substrates suggest that the absolute reactivity was relatively degraded in comparison to the reaction with iodide substrates (Table 2).

The observed highly enhanced catalytic activities of Pd-IPG relative to Pd-rGO can be explained by two possible reasons: (i) the doped ionic polymers on rGO render the system to exhibit good affinity in the aqueous medium, leading to the better dispersion of the Pd-IPG in the reaction medium. As such, the accessibility of substrates and the availability of the catalyst can be substantially enhanced in comparison to those of Pd-rGO. (ii) Upon the basis of the reaction mechanism, the strong cohesion of arylboronic acid and possibly some soluble Pd species detached from the NPs with the long ethylene oxide groups in the ionic polymers can facilitate the effective diffusion of reacting species together in the vicinity of the Pd NPs, leading to the accelerated coupling reaction (Fig. 3). However,

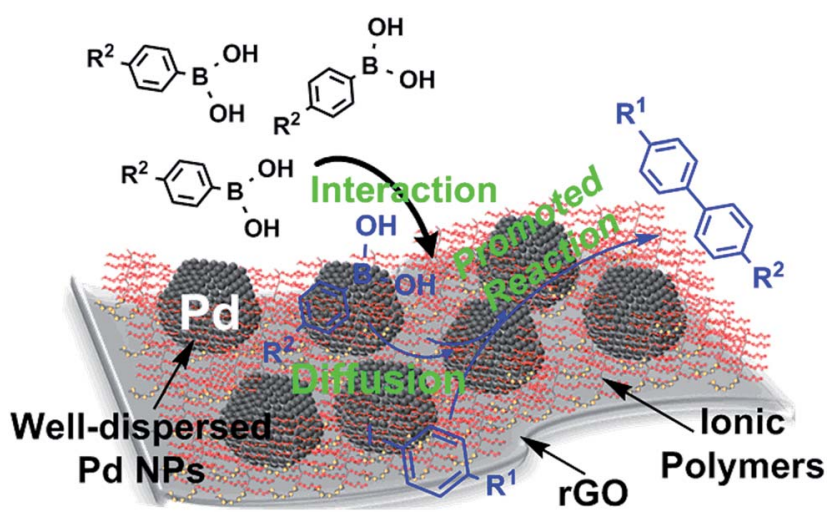

Fig. 3 Schematic illustration of the Pd-IPG nanocomposite catalyst's role on the reaction between aryl iodides and arylboronic acids. the reason for low catalytic activity in the Suzuki coupling reactions with bromide substrates should be addressed and we found that the catalytic activity is considerably governed by an active surface area, which is mainly related to the metal NP size. The report by Mülhaupt et al. provides important information to address the effects of the NP size on the reactivity. ${ }^{\mathbf{1 2}}$ Based on the Mülhaupt's study, we realized that the size effect of Pd NPs is more important than the effect of functionalized supports for enhanced catalytic activity. Furthermore, Mülhaupt's report also expressed that the lager content of oxygenated carbons in the graphene support can promote Suzuki-coupling reactions and this effect corroborated the advantages of the ionic polymer doping in the Pd-rGO catalysts.

Comparative evaluation of the Pd-IPG catalyst was performed in contrast with previously reported, state-of-the-art heterogeneous catalysts for the Suzuki coupling reaction. Table $\mathrm{S} 2 \dagger$ lists the results of the coupling reactions between iodobenzene and phenylboronic acid employing various heterogeneous catalyst systems. Reactions with most of the listed catalysts proceeded with high yields (>90\%). However, in many cases rather harsh reaction conditions were used in comparison to that of Pd-IPG, for example use of toxic solvents (DMF or DME), higher temperature, and longer reaction time. The Pd-IPG exhibited higher catalytic activity (TOF: $990 \mathrm{~h}^{-1}$ ), than the carbon supported Pd catalysts including Pd/SiC-CNT, $\mathrm{Pd} / \mathrm{CNT}$-graphene hydrogel, and $\mathrm{Pd}-\mathrm{Fe}_{3} \mathrm{O}_{4} @ \mathrm{C}$, which exhibited 350,799 , and $324 \mathrm{~h}^{-1}$ TOF values, respectively (Table S2, $\dagger$ entries 6,7 , and 8). ${ }^{13,33,34}$ These results indicate that the Pd-IPG is an effective catalyst with high catalytic activity requiring environmentally clean reaction conditions for Suzuki coupling reactions.

The Pd-IPG catalyst system was then evaluated for repeated use since recyclability of the catalysts is one of the most important issues in viewpoint of cost, and recycling of the PdIPG was investigated for ten repetitions of Suzuki coupling reactions with iodobenzene and phenylboronic acid as model substrates under the optimized conditions. As shown in Table 3 all reactions showed consistently high yields (>96\%). After ten reaction cycles, the recovered Pd-IPG catalyst was characterized by STEM and STEM-EDX, which exhibited same morphology and a high level of retention in the $\mathrm{Pd}$ composition ( $\mathrm{Pd}$ : $47.4 \mathrm{wt} \%$ ) (Fig. S3A and $\mathrm{B} \dagger$ ). For its reliability, further recyclability tests up to 20 cycles were performed. The large retention in the reactivity was observed up to the $14^{\text {th }}$ cycle. However, the reactivity of the Pd-IPG started to decline at the $15^{\text {th }}$ cycle $(89 \%$ yield) and did not recover back to high yield (>95\%) up to the $17^{\text {th }}$ cycle (Table $\mathrm{S} 3 \dagger$ ). When we increased the reaction time

Table 3 Recyclability tests of Pd-IPG for the Suzuki coupling reaction of iodobenzene with phenylboronic acid under optimized conditions ${ }^{a}$

\begin{tabular}{lllllllllll}
\hline Runs & 1st & 2nd & 3 th & 4 th & 5th & 6 th & 7 th & 8 th & 9 th & 10 th \\
Yield (\%) & 99 & 96 & 98 & 97 & 97 & 97 & 98 & 97 & 99 & 98
\end{tabular}

${ }^{a}$ Reaction conditions: Pd-IPG ( $\left.0.1 \mathrm{~mol} \%\right)$, iodobenzene $(0.1 \mathrm{mmol})$, phenylboronic acid $(0.12 \mathrm{mmol})$, base $(0.2 \mathrm{mmol})$, and solvent $(0.8$ $\mathrm{mL}$ ) at $60^{\circ} \mathrm{C}$ under air for $1 \mathrm{~h}$. 
from $1 \mathrm{~h}$ to $1.5 \mathrm{~h}$, the reaction yield went up to $>95 \%$, as shown in Table S3.† The reason why it went up to $99 \%$ is not clear at this point, but the yields at the $18^{\text {th }}$ to $20^{\text {th }}$ recycling were equally high. The fact that there is an 'induction period' at the beginning of each reaction should also be considered. On the basis of STEM studies, the primary reason for the degradation of the catalytic activity was the detrimental change of the PdIPG morphology (Fig. S4 $\dagger$ ). These results indicate that the PdIPG's performance is dependable up to the $14^{\text {th }}$ cycle, presumably owing to the strong interfacial linking ability of the ionic polymers. Furthermore, it should be mentioned that we observed insoluble white salts in the reaction mixture and the content gradually increased with increased repetition (Fig. S3C(ii) $\dagger$ ). This precipitate was analyzed to be sodium iodide (NaI) through STEM-EDX (Fig. S3†). The NaI by-product was readily removed through filtration after dilution with water and the isolated Pd-IPG powder was redispersed in EtOH/ $\mathrm{H}_{2} \mathrm{O}(\mathrm{v} / \mathrm{v}=1: 1)$ for further use.

To explore the Suzuki coupling reaction mechanism employing the Pd-IPG catalyst, a hot-filtration test was performed from the reaction of iodobenzene with phenylboronic acid under the optimized conditions (Fig. S5†). ${ }^{\mathbf{1 6}, 34}$ After $30 \mathrm{~min}$, when the reaction proceeded to give $35 \%$ yield, the reaction mixture was filtered to a preheated vial. The reaction from the filtrate after hot-filtration exhibited no increase in conversion up to $12 \mathrm{~h}$, while the reaction with Pd-IPG continued to achieve 94\% yield after $75 \mathrm{~min}$. This result indicated that the Pd NPs on IPG hardly leached out during the Suzuki coupling reaction. Moreover, the STEM-EDX analysis of Pd-IPG after 10 repeated reaction cycles supported the hot-filtration results. Based upon these findings and our previous mechanistic studies for Pd$\mathrm{Fe}_{3} \mathrm{O}_{4}$ nanocrystal catalyst system, ${ }^{35}$ the Suzuki coupling reaction mechanism in this system with the use of Pd-IPG may be suggested as follows: presumably the Pd species from the PdIPG catalyst system goes into the catalytic cycle of oxidative addition, transmetallation followed by reductive elimination and this active Pd catalyst could be trapped alongside the ionic polymer species, thus allowing for excellent recovery and minimal loss of the catalyst. The observed catalytic efficiency, superior reusability, and chemical stability of the Pd-IPG catalyst suggest that the catalyst could be an effective candidate for constructing biaryl units from $\mathrm{C}-\mathrm{C}$ coupling reactions including Suzuki, Heck, and Sonogashira coupling reactions.

\section{Conclusions}

Pd-IPG nanocomposite catalysts were successfully synthesized through facile one-pot and one-shot-injection methods using BA as a structure-directing agent. The optimized reaction conditions utilizing Pd-IPG as a catalyst for the Suzuki coupling reaction between iodobenzene and phenylboronic acid exhibited excessively high yield (99\%) and catalytic activity (TOF: 990 $\mathrm{h}^{-1}$ ), recording $\sim 16$-fold higher TOF than that of Pd-rGO (TOF: $61.3 \mathrm{~h}^{-1}$ ). This increased reactivity indicates that the presence of ionic polymers on the rGO support can lead to highly facile Suzuki coupling reactions because of great dispersion stability, better accessibility and diffusion of the substrates to the nearby
Pd species and the strong cohesion with the reacting species. However, the reactivity was relatively degraded in the case of the reaction with bromide-mediated substrates. The Pd-IPG catalyst exhibited excellent recyclability with consistently high yields (>96\%) and almost unchanged morphology and $\mathrm{Pd}$ content of the catalyst even after 10 repeated use. Plausible reasons for the retention of $\mathrm{Pd}$ content without noticeable leaching may include the capture of Pd species by nearby ionic polymer groups. These findings suggest that Pd NPs-based catalysts with ionic polymer-doped graphene could be an excellent breakthrough for solving the problems of homogeneous catalysts as well as to significantly enhance the catalytic activity of catalysts based on Pd NPs and carbon supports.

\section{Acknowledgements}

This research was supported by the Mid-Career Researcher Program from the National Research Fund (NRF) grant funded by MSIP, Korea (Project No. NRF-2015R1A2A2A01005965) and partially supported by the Industrial Strategic Technology Development Program funded by the Ministry of Trade, Industry \& Energy (MI), Korea (Project No. 10041850).

\section{References}

1 L. Yin and J. Liebscher, Chem. Rev., 2007, 107, 133-173.

2 N. Miyaura and A. Suzuki, Chem. Rev., 1955, 95, 2457-2483.

3 L. Xue and Z. Lin, Chem. Soc. Rev., 2010, 39, 1692-1705.

4 Q. xiao, S. Sarina, E. jaatinen, J. Jia, D. P. Arnold and H. Zhu, Green Chem., 2014, 16, 4272-4285.

5 A. Fihri, M. Bouhrara, B. Nekoueishahraki, J. M. Basset and V. Polshettiwar, Chem. Soc. Rev., 2011, 40, 5181-5203.

6 B. J. Borah, K. Saikia, P. P. Saikia, N. C. Barua and D. K. Dutta, Catal. Today, 2012, 198, 174-183.

7 R. K. Rai, K. Gupta, S. Behrens, J. Li, Q. Xu and S. K. Singh, ChemCatChem, 2015, 7, 1806-1812.

8 B. Yuan, Y. Pan, Y. Li, B. Yin and H. Jiang, Angew. Chem., Int. Ed., 2010, 49, 4054-4058.

9 Z. J. Wang, S. Ghasimi, K. Landfester and K. A. I. Zhang, Chem. Mater., 2015, 27, 1921-1924.

10 K. Y. Cho, H. Y. Seo, Y. S. Yeom, P. Kumar, A. S. Lee, K. Y. Baek and H. G. Yoon, Carbon, 2016, 105, 340-352.

11 Y. Jang, J. Chung, S. Kim, S. W. Jun, B. H. Kim, D. W. Lee, B. M. Kim and T. Hyeon, Phys. Chem. Chem. Phys., 2011, 13, 2512-2516.

12 G. M. Scheuermann, L. Rumi, P. Steurer, W. Bannwarth and R. Mülhaupt, J. Am. Chem. Soc., 2009, 131, 8262-8270.

13 H. Q. Song, Q. Zhu, X. J. Zheng and X. G. Chen, J. Mater. Chem. A, 2015, 3, 10368-10377.

14 A. Ohtaka, J. M. Sansano, C. Nájera, I. Miguel-García, Á. Berenguer-Murcia and D. Cazorla-Amorós, ChemCatChem, 2015, 7, 1841-1847.

15 S. I. Yamamoto, H. Kinoshita, H. Hashimoto and Y. Nishina, Nanoscale, 2014, 6, 6501-6505.

16 Y. Yang, D. Cong and S. Hao, ChemCatChem, 2016, 8, 900905. 
17 J. Sun, Y. Fu, G. He, X. Sun and X. Wang, Appl. Catal., B, 2015, 165, 661-667.

18 M. Hajighorbani and M. Hekmati, RSC Adv., 2016, 6, 8891688924.

19 Y. Li, X. Fan, J. Qi, J. Ji, S. Wang, G. Zhang and F. Zhang, Nano Res., 2010, 3, 429-437.

20 A. R. Siamaki, A. E. R. S. Khder, V. Abdelsayed, M. S. El-Shall and B. F. Gupton, J. Catal., 2011, 279, 1-11.

21 Z. Zhang, T. Sun, C. Chen, F. Xiao, Z. Gong and S. Wang, ACS Appl. Mater. Interfaces, 2014, 6, 21035-21040.

22 X. Gu, W. Qi, X. Xu, Z. Sun, L. Zhang, W. Liu, X. Pan and D. Su, Nanoscale, 2014, 6, 6609-6616.

23 S. Bai and X. Shen, RSC Adv., 2012, 2, 64-98.

24 V. Georgakilas, M. Otyepka, A. B. Bourlinos, V. Chandra, N. Kim, K. C. Kemp, P. Hobza, R. Zboril and K. S. Kim, Chem. Rev., 2012, 112, 6156-6214.

25 K. Y. Cho, Y. J. Lee, H. J. Kim, H. G. Yoon, S. S. Hwang, Y. K. Han and K. Y. Baek, Polymer, 2015, 77, 55-63.

26 G. Fu, L. Tao, M. Zhang, Y. Chen, Y. Tang, J. Lin and T. Lu, Nanoscale, 2013, 5, 8007-8014.
27 K. Y. Cho, Y. S. Yeom, H. Y. Seo, Y. H. Park, H. N. Jang, K. Y. Baek and H. G. Yoon, ACS Appl. Mater. Interfaces, 2015, 7, 9841-9850.

28 D. S. Su, S. Perathoner and G. Centi, Chem. Rev., 2013, 113, 5782-5816.

29 K. Y. Cho, Y. S. Yeom, H. Y. Seo, P. Kumar, A. S. Lee, K. Y. Baek and H. G. Yoon, J. Mater. Chem. A, 2015, 3, 20471-20476.

30 K. Y. Cho, Y. S. Yeom, H. Y. Seo, P. Kumar, A. S. Lee, K. Y. Baek and H. G. Yoon, ACS Appl. Mater. Interfaces, 2017, 9, 1524-1535.

31 K. Y. Cho, Y. S. Yeom, H. Y. Seo, P. Kumar, K. Y. Baek and H. G. Yoon, J. Mater. Chem. A, 2017, DOI: 10.1039/c6ta09345.

32 R. Narayanan and M. A. El-sayed, J. Phys. Chem. B, 2004, 108, 8572-8580.

33 M. Zhu and G. Diao, J. Phys. Chem. C, 2011, 115, 2474324749.

34 S. P. Andrews, A. F. Stepan, H. Tanaka, S. V. Ley and M. D. Smith, Adv. Synth. Catal., 2005, 347, 647-654.

35 S. Byun, J. Chung, J. Kwon and B. M. Kim, Chem.-Asian J., 2015, 10, 982-988. 